\title{
Hermenéutica, Posmodernidad y Ética Aproximaciones a partir de Gianni Vattimo. ${ }^{1}$
}

Luis Uribe Miranda ${ }^{2}$

"Una noche, me senté a la belleza en las rodillas. Y la hallé amarga. Y la insulté".

(Arthur Rimbaud)

\section{I.- INTRODUCCIÓN}

$\mathrm{E}$ 1 presente artículo manifiesta una exposición. En toda exposición, como tal, se da un exponerse del sujeto. Según esto, el exponerse del sujeto con-siste en "poner-se fuera" de sí. El fuera de sí nos sitúa "más allá del sujeto" o, lo que viene a ser lo mismo, más allá de la modernidad. Por otra parte, este "más allá" no hace referencia a metafísica alguna, sino a un modo de pensar que incluye la diferencia. El "ponernos fuera" de la identidad, como opción del pensar, nos permite decir algo sobre la hermenéutica, la posmodernidad y la ética de un modo "diferente". El pensar del "fuera" de la identidad nos hace transitar por los derroteros de la diferencia.

La diferencia no es una categoría, sino un modo de ser "au-delà de l'essence”, para usar una expresión de Emmanuel Lévinas. Se trata, en cierto sentido, de pensar desde fuera de la modernidad, caracterizado por su proyecto identitario como pre-sencia, pero que no significa una salida de ésta. La no-salida de la modernidad supone transitar por la vía de una concepción no lineal del tiempo y de la historia, dando lugar al

1 Algunas de las ideas desarrolladas en este artículo forman parte del proyecto de investigación "Interpretación del pensamiento y obra del Cardenal Raúl Silva Henríquez, a la luz de los desafíos de una universidad católica en el actual contexto de globalización”, patrocinada por la UCSH durante el año 2000.

2 Magister en Filosofía por la U. de Chile. Académico de la UCSH. 
acontecer del tiempo como temporeidad.

La posmodernidad, como no-salida de la modernidad, propiamente hablando, no es. Es decir, no es algo determinado, no es una mismidad al modo de una presencia objetiva, sino que es un "darse" al modo de una diagnwsiz, de un discernir. Sabemos que esta palabra, posmodernidad, no posee un sentido unívoco. Lo que sí parece claro, es que dice el "darse" de una caducidad, la caducidad de la modernidad fundada en el principio de razón suficiente.

En lo que sigue, tratamos de res-ponder la pregunta por la hermenéutica, la posmodernidad y la ética. Este res-ponder no tiene la pretensión de elevarse como respuesta, sino manifestarse como aproximación. En esto nos dejamos "guiar" en el pensar por el filósofo Italiano Gianni Vattimo.

En lo que respecta a nuestra exposición, hemos dividido nuestra reflexión en cuatro momentos. El primero, sobre la herméneutica en general, a fin de descubrir las relaciones de Vattimo con otros autores. El segundo, sobre la posmodernidad y su vinculación con el "fin de la historia". El tercero, sobre las implicancias de la hermenéutica y la posmodernidad en el plano de la re-flexión ética. El cuarto, y último, trata de plantear algunas consideraciones finales.

\section{II.- La Hermenéutica Filosófica}

\section{II.1.- El comprender como dimensión ontológica:}

La hermenéutica filosófica ha sido considerada, por algunos teóricos, como la filosofía de la época contemporánea. Que esta filosofía se ha vuelto el lenguaje común de la filosofía, significa que ha intentado interpretar la complejidad de problemas que aquejan al hombre contemporáneo.

Esta filosofía surge en el mundo alemán a partir de las reflexiones de Martín Heidegger y proseguidas por Hans-Georg Gadamer, de modo más sistemático, pero es heredera de una gran tradición. Encontramos antecedentes en Aristóteles, en especial en su texto peri ermeneiaz, como 


\section{HERMENÉUTICA, POSMODERNIDAD Y ÉTICA / Uribe Miranda}

en San Agustín, en el texto De Doctrina Christiana. Más aún, recordemos que el cisma de la Iglesia se suscita por un problema hermenéutico relacionado con la interpretación de los textos bíblicos. En la época "moderna", por decir de alguna manera, existen antecedentes en el teólogo luterano Schleiermacher, pero sobre todo, en el filósofo historicista Wilhelm Dilthey, el cual va a poner el acento en la crítica al positivismo científico haciendo la distinción entre las Geisteswissenchaften, en contraposición a las Naturwissenchaften. Se desliza aquí la discusión sobre la Erlebnis. La Erlebnis, como vivencia, sólo será posible en la medida que aceptemos la existencia de la vida como elemento digno de ser "sabido". Así, las vivencias, como producciones del espíritu, dan lugar a una nueva manera de entender la ciencia, dando lugar a las ciencias sociales. También podemos rastrear elementos en Edmund Husserl, sobre todo en el concepto de Lebenswelt. El mundo de la vida será la condición de posibilidad para re-flexionar sobre las vivencias pero como "hechos de conciencia”. Más contemporáneamente, será Heidegger, en múltiples obras, el que dará un giro en el modo de reflexionar sobre el carácter ontológico del comprender y del interpretar, dado que el hombre poseería una estructura ontológica circular y lingüística. Será en esta tradición en la que se insertarán las reflexiones de Gadamer y Vattimo.

Para el filósofo Gianni Vattimo, la hermenéutica es "la filosofía que se desarrolla en el eje Heidegger-Gadamer. En la gama de los problemas y soluciones que elaboran estos autores se pueden colocar coherentemente todos los aspectos y las diferentes vías seguidas por la hermenéutica en el curso de nuestro siglo... Vista así, la hermenéutica revela sus dos aspectos constitutivos: el de la ontología y el de la Sprachlichkeit, la lingüisticidad"3. Es decir, los dos elementos enunciados por Vattimo, la ontología y la lingüisticidad, comportan la identidad de la reflexión hermenéutica.

El primero, desprendido del planteamiento que hace Martín Heidegger a partir de Sein und Zeit, donde es necesario realizar la pregunta que interroga por el sentido del ser ${ }^{4}$. Recordemos que esta pregunta no se puede homologar a un modo de aprehensión intelectual, sino que com-

3 VATTIMO, Gianni; Más allá de la interpretación, Ed., Paidós, Barcelona, 1995, Pág. 39.

4 Cfr. HEIDEGGER, Martín; Ser y tiempo, Ed., Universitaria, Santiago, 1997. En especial, el parágrafo 
porta un análisis de la estructura ontológica del ser humano. De hecho, para Heidegger, la pregunta cómo buscar, no consiste sólo en preguntar "por", sino en preguntar "a". Es decir, se hace necesario ingresar en el circuito del preguntar. En un texto posterior a Sein und Zeit, Heidegger planteará que toda pregunta comporta dos elementos. "En primer lugar, toda pregunta metafísica abarca íntegro el problematismo de la metafísica. Es siempre el todo de la metafísica. En segundo lugar, ninguna pregunta metafísica puede ser formulada sin que el interrogador, en cuanto tal, se encuentre dentro de ella, es decir, sin que vaya él mismo envuelto en ella" . Esto es que no podemos preguntar sin estar completamente involucrado con lo que preguntamos, de tal suerte que esta pregunta nos patentiza nuestro modo de ser: somos comprensión.

La frase antes planteada es una tesis fundamental de la hermenéutica. Heidegger, en los parágrafos 31, 32, 33, 34 y 35 de Sein und Zeit, desarrolla ampliamente el tema de la comprensión como existenciario del dasein y su vinculación con la interpretación y el lenguaje. Para efectos de la presente reflexión sólo diremos que el filósofo de Friburgo plantea que la Verstehen es Lichtung. Esto es, que la comprensión es un modo de ser del dasein y ésta consiste en un "estado" de apertura que coincide con la existencia ${ }^{6}$. Dicha co-incidencia no podemos entenderla como adaequatio, sino como pertenencia. Por otra parte, este "estado" de apertura, que es la comprensión, se vive como interpretación. Por lo mismo, la interpretación en Heidegger es el despliegue de la comprensión, es el "estar-siendo" del dasein. Por lo tanto, la afirmación de Vattimo hace referencia a este elemento constitutivo de la hermenéutica que es la ontología.

\section{II.2.- El lenguaje como modo de ser:}

El segundo elemento hace referencia a la reflexión de Gadamer. Para Vattimo el elemento de la linguíísticidad sería un aporte de Gadamer. Sin embargo, y contraviniendo en parte la tesis de Vattimo, creemos

5 HEIDEGGER, Martín; ¿Qué es metafísica?, Ed., Fausto, Buenos Aires, 1992, pág. 39.

6 Recordemos que Heidegger plantea que el dasein es la esencia de la existencia humana. Además, que la palabra existencia en un sentido etimológico significa ser desde fuera, haciendo justamente referencia a una apertura ontológica. Esto es lo que queremos decir con la expresión Lichtung, aún sabiendo que ésta designa el "claro del sol". 


\section{HERMENÉUTICA, POSMODERNIDAD Y ÉTICA / Uribe Miranda}

que este elemento ya aparece en Heidegger. En muchos parágrafos de Sein und Zeit, pero sobre todo en obras posteriores, hay referencias a la lingüísticidad del hombre. En la carta dirigida a Jean Beaufret, el filósofo de Friburgo nos dona su palabra: "El lenguaje es la casa del ser. En su vivienda mora el hombre" y agregará en otros párrafos que "el hombre no es un ser viviente que junto con otras facultades posee también el lenguaje. Más bien es el lenguaje, la casa del ser en la que el hombre, morando, ec-siste, en cuanto guardando esta verdad, pertenece a la verdad del ser". . Esto es, que la lingüísticidad del ser del hombre no es una determinación posterior, al modo de un acto segundo, de la existencia. Más aún, es un modo de habitar. El habitar como morar hace referencia al sentido primario de la voz griega hqoz, que como sabemos está a la base de ética. Es decir, que el lenguaje pone en relación al hombre con el ser, el mundo, la verdad y la ética. Es aquí, donde se juega la cuestión del "sentido" del ser del dasein".

Por otra parte, al enunciar el primer elemento identitario de la hermenéutica según Vattimo, dejamos tras-lucir que el ser del hombre es comprensión y que vive como interpretación pero, además, que su estructura ontológica es circular. Esto viene a patentizar la existencia del círculo de la comprensión, pero a la vez, muestra el nexo entre Heidegger y Gadamer. Para este último, "Heidegger hace una descripción fenomenológica plenamente correcta cuando descubre en la presente "lectura" de lo que "hay" la preestructura de la comprensión. En Ser y tiempo concretó el tema general, formulado por él como problema hermenéutico, en la pregunta por el ser" 10 y agrega, "el círculo hermenéutico adquiere así en el análisis de Heidegger un nuevo significado... reconoce, en cambio, que la comprensión del texto está determinada permanentemente por el movimiento anticipatorio de la precomprensión. Lo que Heidegger describe así no es sino la tarea de concreción de la conciencia histórica" ${ }^{11}$. Sin embargo, Gadamer considera que esto no es suficiente. Es necesario agregar

7 HEIDEGGER, Martín; Carta sobre el humanismo, Ed., Taurus, Madrid 1959, Pág. 7.

8 Ibidem, Pág. 30-31.

9 Cfr. HEIDEGGER, Martín; "Hölderlin y la esencia de la poesía” en Arte y poesía, Ed., F.C.E., México, 1995. En especial las páginas que van desde la 132 hasta la 148, dado que es aquí donde Heidegger plantea que el hombre es lenguaje, que es un modo de ser del existente que se transparenta en el habla.

10 GADAMER, Hans-Georg; Verdad y método II, Ed., Sígueme, Salamanca, 1992, Pág. 66.

11 Ibidem, Pág. 67. 
otros elementos. El primero que aparece es el "anticipo de compleción". "Según él, sólo es comprensible lo que constituye realmente una unidad de sentido acabada... no se presupone sólo una unidad de sentido inmanente que orienta al lector, sino que la comprensión de éste es guiada constantemente por expectativas trascendentes que derivan de la relación con la verdad del contenido"12. Según estos párrafos, nos parece "claro" que Gadamer toma distancia de Heidegger. Esta distancia consiste en comprender de modo diferente la pre-comprensión, lo que para Heidegger es el "estado" de apertura en el tiempo, Gadamer lo concibe como un anticiparse a la comprensión. Este anticiparse, que denomina de compleción, tiene significado en la unidad de sentido y contenido. Es decir, lo que queremos comprender debe ser ya una unidad de sentido, sin esto no es posible arribar a una comprensión verdadera. Pero además, incorpora las expectativas del sujeto, que Gadamer denomina "expectativas trascendentes", a fin de incorporar elementos de la conciencia o "hechos de conciencia", como diría Husserl. Nos parece, sin embargo, que Gadamer pretende objetivar el círculo de la comprensión a partir de la unidad de sentido y contenido, al modo de cierto "positivismo lógico".

Sin contravenir lo anterior, y dando cuenta de nuestra concordancia parcial con la tesis de Vattimo, nos parece que Gadamer incorpora el elemento de la lingüísticidad a la hermenéutica. Esto queda de manifiesto en la asunción de los postulados de Aristóteles. Para éste, el hombre es un ser dotado de logoz, nos recuerda Gadamer en Verdad y método. Dicho enunciado significa que "el hombre es el ser vivo dotado de lenguaje"13. Por lo mismo, no podemos pensar que el lenguaje sea sólo un medio de comunicación. Gadamer dirá que "el lenguaje no es un medio más que la conciencia utiliza para comunicarse con el mundo. No es un tercer instrumento al lado del signo y la herramienta que pertenecen también a la definición esencial del hombre. El lenguaje no es un medio ni una herramienta" 14 . Que el lenguaje no sea un medio ni una herramienta significa aquí, por de pronto, que no es algo que se pueda usar y des-usar, tomar y dejar a voluntad. El lenguaje no afinca en el hombre por voluntad propia, sino que es algo que recibe. Gadamer usa la palabra "dotado", es 


\section{HERMENÉUTICA, POSMODERNIDAD Y ÉTICA / Uribe Miranda}

decir, que el lenguaje no le pertenece de antemano al hombre, sino que lo recibe como un "don", un "regalo". He aquí la gran paradoja. Lo que define el ser del hombre no le pertenece. Que no le pertenezca significa que no lo tiene "dado" o, lo que viene a ser lo mismo, que la esencia del hombre es aquello que todavía no es; es un proyecto que articula sentido. Es por esta razón que Gadamer dirá: "El lenguaje es así el verdadero centro del ser humano... El hombre es realmente, como dijo Aristóteles, el ser dotado de lenguaje. Todo lo humano debemos hacerlo pasar por el lenguaje" 15 .

En esta misma línea, digamos que "la hermenéutica no constituye un método determinado que pudiera caracterizar por ejemplo a un grupo de disciplinas científicas frente a las ciencias naturales. La hermenéutica se refiere más bien a todo el ámbito de la comunicación intrahumana" ${ }^{16}$. Es decir, la comunicación intrahumana sólo es posible por el lenguaje y éste posibilita el hecho de que la hermenéutica no sea sólo un método, sino más bien, una filosofía de la vida como lenguaje. En este sentido, podemos plantear que se centra en las vivencias humanas. Será la Erlebnis el motor del quehacer hermenéutico de Gadamer y la tradición en la que se inserta.

Sin embargo, nos parece necesario reflexionar brevemente sobre la relación entre hermenéutica e historicidad a fin de esclarecer, someramente, el "darse" de la posmodernidad. Para los pensadores hermenéuticos, la posmodernidad hace referencia al fin de la historia, por cuanto implica la caída de un tipo de racionalidad y, por ende, de un tipo de metafísica. En este sentido, nos parece pertinente presentar la re-flexión del filósofo de Turín Gianni Vattimo, como un exponente relevante de la hermenéutica contemporánea y que constituye el eje de nuestra reflexión.

\section{III.- Posmodernidad Como Fin de la Historia}

Para Gianni Vattimo la posmodernidad se vincula con el fin de la historia. En efecto, "las caracterizaciones más ampliamente aceptadas de la posmodernidad sea aquella que la presenta como el fin de la historia"17. 
El carácter apocalíptico que podría tener esta manera de comprender la historia, de la cual nos advierte Vattimo, no pretende expresar el fin de la historia como el no acontecer nada, sino que se vincula con la caída de los llamados "grand récits". La caducidad de estos discursos globales provienen de un conflicto de racionalidades o, si se quiere, de la "debilidad" de la razón para articular un discurso capaz de comprender el devenir de la historia que, a su vez, lleva aparejado el caminar de la humanidad. Esto es, por lo pronto, que la historia de la humanidad ha querido transitar hacia el logro de la emancipación. En este sentido, la historia sería historia de la emancipación de la humanidad. Sin embargo, para Vattimo, y también para Lyotard, la caducidad de la historia se ve expresada por acontecimientos en los que la humanidad, lejos de transitar hacia la emancipación, ha caído en la esclavitud. Acontecimientos como Auschwitz, los detenidos desaparecidos y las sistemáticas violaciones a los derechos humanos acontecidas durante la dictadura de Pinochet en Chile, no han hecho otra cosa que dar cuenta, des-enmascarar, la insuficiencia y debilidad de la racionalidad historicista para determinar, de manera global, el curso de la humanidad a partir de una concepción progresista de la historia. Pero, además, vienen a poner de manifiesto, por un lado, que un discurso global sobre la historia no es más que una "expresión de la violencia ideológica" 18 y, por otro, que el proyecto moderno ha fracasado en su afán de lograr la emancipación de la humanidad.

La reflexión Vattimiana nos lleva a plantear que la historia no tendría un sentido teleológico. La historia no tiene un fin y pide ser reflexionada como un final. Reflexionar la historia como un final implica asumir el final de la modernidad. Para Vattimo "pensar lo posmoderno como fin de la historia, como el final del fin, no significa, entonces, darse cuenta de que la cuestión hubiera ya dejado de proponerse, sino, al revés, situar en el primer plano de una atención central la cuestión de la historia como raíz de legitimaciones" ${ }^{19}$. Es decir, lo que ha llegado a su fin es la legitimidad de los "meta-relatos" historicistas modernos, por cuanto éstos serían expresión de una "metafísica de la voluntad de poder" que concibe la historia de modo lineal. Según esto, "la modernidad es la época de la legitimación metafísico-historicista, la posmodernidad es la puesta en

17 VATTIMO, Gianni; Ética de la Interpretación, Ed. Paidós, Argentina, 1992, Pág. 15.

18 Ibidem, Pág. 17.

19 Ibidem, Pág. 20. 


\section{HERMENÉUTICA, POSMODERNIDAD Y ÉTICA / Uribe Miranda}

cuestión explícita de este modo de ligitimación" ${ }^{20}$. Por consiguiente, la posmodernidad como fin de la historia no puede comprenderse como algo que viene después de la modernidad, cuyo fin sería rectificar y corregir la modernidad, sino que es hacerse la pregunta por la legitimidad del discurso moderno al interior de la modernidad, a la vez que hacerse cargo y tematizar los problemas que se derivan de la disolución de dicho discurso.

La vía que nos propone Vattimo, para reflexionar la posmodernidad como fin de la historia y haciéndonos cargo de lo que se deriva de esta disolución, es el camino mostrado por Heidegger con respecto a la metafísica. Esto es, que la metafísica no puede ser superada "como si se tratara de un error del que uno pudiera liberarse mediante la crítica" ${ }^{21} \mathrm{O}$, lo que viene a ser lo mismo, que la metafísica no puede ser superada críticamente. La imposibilidad de la superación significa, entonces, asumir la des-fundamentación de la historia. La des-fundamentación de la historia es lo que Vattimo llama fin de la historia. Esto no significa la ausencia total de la historia, sino que la historia no tiene un fin único y estable a través de las diferentes épocas, donde cada una de esas épocas es expresión de una metafísica ${ }^{22}$. Precisando aún más esta reflexión Vattimiana, podemos decir que cada época histórica tiene un fundamento (Grund), pero no de una manera estable, sino esencialmente móvil. Alguien podría objetar diciendo que un fundamento móvil, que va teniendo diversas configuraciones, no fundamenta nada. Sin embargo, esto es precisamente lo que está planteando Vattimo. Es decir, "no hay" un fundamento único y "fuerte" capaz de dar cuenta, de una vez y para siempre, de modo legítimo, del curso de la historia. El fundamento de la historia, tal y como nos lo plantea nuestro autor, es des-fundamento. Ser des-fundamento no es la ausencia de fundamento sino su presencia. El presenciar del fundamento en el des-fundamento es la movilidad propia del acontecer de la historia.

La posmodernidad comprendida como fin de la historia no podría, entonces, ser entendida como una decadencia. Para Vattimo, "el final de los "meta-

20 Idem.

21 Ibidem, Pág. 24.

22 Esta comprensión de la metafísica como historia del Ser y construcción de una época, aparece de modo manifiesto en las primeras líneas del texto de Martín Heidegger "La época de la imagen de mundo"contenido en el libro Caminos de bosque publicado en la editorial Alianza/Universidad. 
relatos" pensado desde el horizonte de la historia de la metafísica y de su disolución..., es el darse del ser en la forma de la disolución, del debilitamiento y de la mortalidad, pero no de la decadencia, porque no hay ninguna estructura superior, fija o ideal, con respecto a la cual la historia hubiera decaído"23. Es decir, comprender la posmodernidad como decadencia es comprenderla desde un fundamento estable y es esto, justamente, lo que Vattimo no plantea. Por otra parte, reflexionar sobre el fin de la historia permite re-cuperar el pasado como presente. Esto es, que al no tener la historia un sentido teleológico, un progreso ilimitado, podemos tomar el pasado como pertenencia a una tradición, a una memoria, a una cultura y, por ende, presente en la "historia-presente". Pensar la historia sin un fin exige salir de la racionalidad tecno-cientifica y transitar hacia lo que Heidegger llamaba "pensamiento rememorador" o Andenken.

La historia es acontecer y no historicismo. Es aquella que manifiesta la presencia de la posmodernidad en la modernidad. La historia es lo que manifiesta la des-fundamentación como fin de la historia y que exige un giro en la racionalidad a fin de re-pensar las consecuencias de dicho giro en todas las esferas del habitar humano. En otras palabras, se trata de asumir la no existencia de "hechos" al modo de una supuesta objetividad, capaz de obligar a la memoria, la cultura y la razón. No hay razón suficiente que justifique el no-logro del progreso. La creciente pobreza, el desastre ecológico, las violaciones a los derechos humanos, los regímenes autoritarios, la educación ideológica y la creciente moralización no tienen fundamento, ni mucho menos "razón de ser".

\section{IV.- Hermenéutica, Posmodernidad y Ética}

La re-articulación de la racionalidad hermenéutica exige re-pensar los diversos sectores de su aplicación. Entre estos sectores se encuentra la ética. La posmodernidad como caída de los "grands récits", disolución del sujeto y fin de la historia, como discurso emancipatorio, nos permiten afirmar que asistimos a una re-habilitación de la "filosofía práctica".

Recordemos que Nietzsche, en La Gaya Ciencia, había profetizado sobre el advenir del nihilismo. La frase del loco que anuncia que ¡Dios 


\section{HERMENÉUTICA, POSMODERNIDAD Y ÉTICA / Uribe Miranda}

ha muerto $;$, interpretado por algunos como un abierto ateísmo, no es más que la creciente preocupación por la des-fundamentación de la moral. Es decir, la moral no tiene principios justificados metafísicamente, tanto en el sentido medieval como moderno. En este sentido, una reflexión ética contemporánea no puede desconocer la reflexión nietzsheana, pues ésta ya es una crítica a la modernidad y a la insuficiencia de la razón científica. La asunción del nihilismo, en esta re-flexión, tiene un sentido de re-cuperación, un volver a dar lugar a la cuestión de los valores. El nihilismo, como una trans-valoración de los valores, de la ética y del hombre en su multiculturalidad, es un pretexto hermenéutico. En otras palabras, el nihilismo contemporáneo es un pre-texto para reflexionar desde la hermenéutica sobre la ética y sus diferentes contenidos trastrocados por el impacto de la tecno-ciencia moderna. Esta será la vía abierta por Vattimo, por cuanto su re-flexión ética transitará por los derroteros del nihilismo de Nietzsche, la ontología de Heidegger y la hermenéutica de Gadamer.

\section{IV.1.- Ética de la Interpretación}

La ética contemporánea exige ser reflexionada desde la caída de la modernidad. Para el filósofo de Turín, la hermenéutica tiene una invocación a ser ética "por cuanto hace valer la instancia ética como elemento determinante de su crítica a la metafísica tradicional, y a su última encarnación representada por el cientifismo" 24 . Según esto lo que permite el "salto" de la metafísica tradicional y el cientifismo es la ética, por cuanto ésta "en cuanto ethos, costumbres, cultura compartida de una época y una sociedad, aquello que, en última instancia, "desmiente" al científico y su característica pretensión de reducir la verdad únicamente a los enunciados experimentales comprobados por el proceder metódico de la ciencia matemática de la naturaleza" 25 . En este sentido, la ética es la vía de crítica al pensamiento moderno y su afán de ser verdad universal ${ }^{26}$ al mismo tiempo, que permite la " rehabilitación de la filosofía práctica" en el pensamiento contemporáneo.

24 VATTIMO, Gianni; Ética de la interpretación, Ed., Paidós, Argentina, 1992, Pág. 205.

25 Ibidem, Pág. 206.

26 Cfr. VATTIMO, Gianni; Más allá de la interpretación. En especial el capítulo segundo titulado "Ciencia" y el apéndice 1 "La verdad de la hermenéutica". 
Para la Hermenéutica, la ética no puede ser normativa, al modo de existir una verdad única que determine lo bueno y lo malo, sino de bienes. En efecto, dirá Vattimo, siguiendo a Scheleiermacher, "La ética que la hermenéutica hace posible parece ser principalmente una ética de los bienes... y no una ética de los imperativos. O mejor, si algún imperativo se delinea en ella es el que prescribe reconducir, entendiendo la interpretación como acto de traducción... los varios logoi-discursos de los lenguajes especializados, así como las diversas esferas de intereses y ámbitos de racionalidad autónomos - al logos - conciencia común, al sustrato rector de los valores compartidos por una comunidad histórica viviente que se expresa en su lengua ${ }^{27}$. Esto es, que la ética hermenéutica o de la interpretación pretende no caer en los imperativos, propios del Kantismo, a través de la recuperación del lenguaje, en cuanto discurso perteneciente a una determinada tradición cultural, los cuales no se pueden definir, ni menos interpretar, rígidamente. Cada discurso posee un núcleo valorativo, propio de cada cultura, que se ve articulado en la historia. La historicidad, será un factor determinante en la ética de la interpretación, por cuanto los discursos éticos serán, también, históricos al igual que los valores. En efecto, para Vattimo, " los valores a partir de los cuales se opera en cada ocasión dentro del diálogo social la preferibilidad de cierta opción, son valores radicalmente históricos" ${ }^{28}$, y en consecuencia, susceptibles de ser interpretados desde la temporalidad histórica.

La ética de la interpretación, así entendida, pretende desligarse de una normatividad ética, por cuanto su "fundamento" histórico trasciende a la ética de la comunicación, que pretende un "a priori de la comunidad" universal, donde el "sujeto fuerte" prescribe los imperativos. Según Vattimo, la ética de la comunicación, inspirada en el transcendentalismo Kantiano, es una "restauración de la metafísica: ello no sólo y hasta un tanto porque el sujeto cuya transparencia se trata de promover... se piense a partir del modelo de sujeto propio de la metafísica y del cientificismo moderno, es decir, como tendencialmente resuelto en autoconciencia, sino también y sobre todo porque el ideal normativo de la comunicación ilimitada apoya su categoricidad sobre la base de asumir una estructura esencial, que

27 VATTIMO, Gianni; Ética de la interpretación, Pág. 207.

28 Ibidem, Pág. 209. 


\section{HERMENÉUTICA, POSMODERNIDAD Y ÉTICA / Uribe Miranda}

siendo rectora para toda experiencia histórica, se sustrae ella misma, sin embargo, al devenir"29. Luego, en una primera aproximación, la ética de la interpretación viene a tomar distancia de la ética de la comunicación, en especial de Habermas, por cuanto asume el devenir histórico que nos revela la caída de la modernidad y de la metafísica. EL sujeto ético ya no será "fuerte",sino más propia y originariamente "débil" (debole). El "sujeto débil" es el de la era sin metafísica y posmoderno, es, en términos Heideggerianos, el del "ocaso del ser", o mejor, del "ser ocaso". "Ser ocaso" es ser finito, de tal suerte, que el "sujeto débil" es, también finito por tener una existencia histórica marcada por el devenir y la finitud ${ }^{30}$. Por tanto, para Vattimo, la ética de la comunicación es ahistórica y no cumple como salida ética, de la metafísica y el cientifismo, posibilidad que sí tendría la hermenéutica.

La ética de la interpretación es la que hace valer la historicidad como "pertenencia" a una tradición, donde la verdad no es entendida como absoluta. "La experiencia de la verdad no acaece en el reflejo del objeto por parte de un sujeto que hubiera de tornarse transparente, sino como articulación - o interpretación - de una tradición (una lengua, una cultura) a la que la existencia pertenece y a la que reformula enviando nuevos mensajes a los demás interlocutores. Eticidad e historicidad, aquí, coinciden" 11 , como eventos de destino, donde los valores son reconocidos históricamente. El ser eventos de destino, de la ética de la interpretación, es reconocer su constitución nihilista. Esto es, que en el destino, "que es el de la época del final de la metafísica, en la cual, tal como dice Heidegger, se ilumina un primer relampaguear del evento del ser, por cuanto, en ese momento, hombre y ser pierden las características que la metafísica (el pensamiento de la presencia, de la objetividad, o de la voluntad de poder) les había asignado"32. Según esto, la ética de la interpretación no tiene un fundamento metafísico, donde los valores y la verdad no son adecuaciones, sino interpretaciones y esto es posible porque habitamos en un mundo regido por los "mass media"; somos, como dice Vattimo, "una sociedad transparente", donde ya no hay verdad, ni discursos globales, sino interpretaciones históricamente pertenecientes.

29 Ibidem, Pág. 211.

30 Sobre la relación entre historia y finitud se puede consultar HEIDEGGER, Martín; Ser y tiempo, en especial, los parágrafos 65 y 74, dada la vinculación entre Heidegger y Vattimo.

31 VATTIMO, Gianni; Ética de la interpretación, Pág. 215.

32 Ibidem, Pág. 217. 
Es por tanto, una ética del fin de la metafísica y, por ende, del cumplimiento de la modernidad, es decir, del nihilismo. Como bien afirmará Vattimo: "reconociendo su propio destino nihilista, la hermenéutica da lugar a una ética que tiene en su base una ontología de la reducción ${ }^{33}$ y del aligeramiento; o si se quiere, de la disolución"34.

La ética de la interpretación surge por el fin de la modernidad. Es decir, como una no-salida de la modernidad, como una ética de la diferencia.

\section{V.- Consideraciones Finales...}

Nuestra re-flexión no pretendía dar res-puesta, sino sólo res-ponder. El pretender ser res-puesta nos situaría en el marco de una metafísica moderna, en cambio, transitar por el res-ponder nos encamina a una re-flexión que pretende ponderar los alcances de una reflexión posmoderna, al modo de Gianni Vattimo, que se puede aplicar a los diversos ámbitos del habitar humano.

El epígrafe de Rimbaud ${ }^{35}$, con el que comenzamos nuestra re-flexión, hace referencia a la caída de la noción de belleza, una belleza que ya no es "dulce" y que puede ser "insultada" dado que ya no posee un fundamento metafísico que justifique su "dulzura" y "honorabilidad".

Recordemos que la belleza, al igual que la verdad, son comprendidos por la metafísica tradicional como "trascendentales del ente". En este sentido, la belleza y el ente se convierten. Que se convierten, significa que todo ente es bello en sí mismo, dando lugar a una belleza también en sí misma, que se manifiesta como una realidad objetiva. Es a esta realidad objetiva de la que la posmodernidad, al igual que Rimbaud en su tiempo, pretende salir.

Las implicancias de tal afirmación se presenta como radical. Es decir, que viene a plantear que los fundamentos de la modernidad se sostienen

33 Cfr. VATTIMO, Gianni; Más allá del sujeto, Ed., Paidós, Barcelona, 1992. En especial el capítulo II, "Hacia una ontología del declinar".

34 VATTIMO, Gianni; Ética de la interpretación, Pág. 222.

35 Cfr. RIMBAUD, Arthur; Una temporada en el infierno, Ed., Mondadori, Madrid, 1991, Pág. 3. 


\section{HERMENÉUTICA, POSMODERNIDAD Y ÉTICA / Uribe Miranda}

en la subjetividad de la conciencia. La conciencia de la realidad no es la realidad. La frase "ya no hay hechos, sólo interpretaciones", en cuanto des-fundamento de toda hermenéutica, se presencia como "posibilidad posible" de decir, con sentido, algo sobre la realidad que ha salido de la "objetividad de los hechos". El salir de la objetividad de la realidad, nos hace plantear la pregunta por la legitimidad de los discursos metodológicos, fundados en el positivismo y constructivismo, que pretenden "intervenir" la realidad. El inter-venir es una manera de construir, modelar y reflejar la realidad sobre la base de la relación "sujeto-objeto". Sin embargo, esta relación lineal ha sido confutada por la misma modernidad y exige ser reflexionada desde la diferencia.

La diferencia reflexionada por Vattimo nos exige pensar la realidad como co-pertenencia de identidad y diferencia. Esto implica que la noción de "lo social" como conglomerado homogéneo no tiene fundamento, por cuanto esta noción se gatilla a partir de la "metafísica de la voluntad de poder", cuya mayor expresión las encontramos en la técnica e ideologías modernas. Se requiere, por ende, saltar de la identidad pero sin salir de ésta, de tal manera que sólo podemos pensar la identidad social a partir de la diferencia social. Reflexionar lo social a partir de la diferencia es una tarea "pendiente" que exige el "olvido del ser", el "olvido del ser racional" y el "olvido de la modernidad". Se trata, por tanto, de una Verwimdung, de una superación, que no es homologable a una superación dialéctica, de los rasgos de la modernidad, es decir, de un asumir los planteamientos de la hermenéutica filosófica que reflexiona sobre la posmodernidad y sus alcances en la ética.

La re-flexión aquí realizada, sobre la hermenéutica, la posmodernidad y la ética, es una aproximación a partir de Gianni Vattimo. Como tal, y consecuente con lo planteado, no tiene como finalidad levantarse como única vía, como única verdad, sino que pretende ser un "aporte" al pensar en la época contemporánea. La re-flexión como tal, y eso es lo que pretende el guión que separa la palabra, es un volver. En este sentido, toda re-flexión vuelve...

Punta Arenas, Enero del 2001. 